\title{
Bifurcations in Globally Coupled Chaotic Maps
}

\author{
Satoru Morita* \\ Department of Physics, Graduate School of Sciences, \\ Kyoto University, Kyoto 606, Japan
}

\begin{abstract}
We propose a new method to investigate collective behavior in a network of globally coupled chaotic elements generated by a tent map. In the limit of large system size, the dynamics is described with the nonlinear FrobeniusPerron equation. This equation can be transformed into a simple form by making use of the piecewise linear nature of the individual map. Our method is applied successfully to the analyses of stability of collective stationary states and their bifurcations.
\end{abstract}

PACS: 05.45.+b, 05.70.Ln, 82.40.Bj

Keyword: globally coupled maps, chaos, Frobenius-Perron equation, bifurcation, collective behavior

Typeset using REVTEX

*E-mail address: morita@ton.scphys.kyoto-u.ac.jp. 


\section{INTRODUCTION}

Globally coupled dynamical systems form an important class of models in nonlinear dynamics. The all-to-all nature of the coupling may be regarded as an idealization of longrange coupling or an approximation to a short-range coupling high-dimensional lattice. Such systems are useful in modeling diverse phenomena such as Josephson junction arrays [1], multimode laser [2], charge-density wave [3], evolutionary dynamics [4], biological information processing and neurodynamics $[5$ 8 8 . A simplifying assumption is often made that the coupling strength is uniform over all coupled pairs. This makes the theoretical treatment much easier compared with systems with short-range interaction [9].

In the present paper, we study globally coupled maps (GCM) introduced by Kaneko [12]. They form a dynamical system of $N$ local mappings which are under a common internal field. The system is thus a mean-field version of coupled map lattices (CML) [10,11]. The explicit form of GCM we will work with is given by

$$
x_{n+1}(i)=(1-\epsilon) f\left(x_{n}(i)\right)+\frac{\epsilon}{N} \sum_{i^{\prime}=1}^{N} f\left(x_{n}\left(i^{\prime}\right)\right) .
$$

Here $n$ represents a discrete time step, $i$ the index of the elements $(i=1,2,3, \ldots, N)$, and $\epsilon$ the coupling constant. It is assumed that the map $f(x)$ gives rise to chaotic dynamics. We specifically consider a tent map

$$
f(x)=1-a|x|
$$

with parameter $a$. The piesewise linearity of the map simplifies the analysis considerably as we see later. The range of $x$ of each map is given by $[-1,1]$. The coupling is only through the mean field

$$
h_{n}=\frac{1}{N} \sum_{i=1}^{N} f\left(x_{n}(i)\right) .
$$

There are a great variety of phenomena generated by such globally coupled maps, and

they seem to be due to two conflicting natures involved in the dynamics [12]. On one side, the presence of a common driving force coming from the mean field favors mutual 
synchronization. On the other side, unstable growth of the difference in $x_{n}$ between any pair of elements tends to destroy such synchrony. If the coupling constant $\epsilon$ is sufficiently large, mutual synchronization will be complete, all elements behaving identically. Then the collective motion is described simply by the single map $f(x)$. On the contrary, if $\epsilon$ is

sufficiently small, the system will be "turbulent" where the elements are scattered and behave chaotically in time. In the present model there also appear band structures for intermediate coupling strengths. The state of perfect coherence loses stability at $a(1-e)=2$, while the transition between turbulent and two-band states occurs at $a(1-e)=\sqrt{2}$. A phase diagram was obtained by Kaneko [13].

In this paper, we shall confine our analysis to turbulent and two-band states. The limit of large $N$ is of our particular concern. Some unexpected features of collective quantities such as the mean field have been found recently. For instance, the mean square deviation of such quantities dose not tend to zero as $N \rightarrow \infty$ but saturates to a finite value. This suggests the existence of nontrivial collective behavior [13 20].

\section{NONLINEAR FROBENIUS-PERRON EQUATION}

For a very large system size, direct numerical simulation of (1) requires enormous computer capacity. Moreover, the occurrence of long transients may complicate the analysis of stationary states and other asymptotic states. For these reasons, an alternative approach seems desirable.

Statistical states of an ensemble of maps governed by (11) can be characterized by its density distribution

$$
\rho_{n}(x)=\frac{1}{N} \sum_{i=1}^{N} \delta\left(x-x_{n}(i)\right),
$$

where $\delta(x)$ is Dirac's delta function. The evolution of $\rho_{n}(x)$ obeys the Frobenius-Perron equation 14, 15,

$$
\rho_{n+1}(x)=\int \delta\left(x-F_{n}(y)\right) \rho_{n}(y) d y
$$


where the effective map $F_{n}(x)$ depends on the mean field $h_{n}$ through

$$
F_{n}(x)=(1-\epsilon)(1-a|x|)+\epsilon h_{n}
$$

The mean field $h_{n}$ is calculated from the density (雨) as

$$
h_{n}=\int f(x) \rho_{n}(x) d x
$$

Equation (5) is called the nonlinear Frobenius-Perron equation (NFPE) [15]. In the limit of large system size the density (团) becomes a "sufficient continuous" function. NFPE is completely analogous to the nonlinear Fokker-Planck equation. In the following, we assume that the initial condition is given by a uniform distribution $\rho_{0}(x)=\frac{1}{2}$, that is, the elements of the original GCM are distributed in a completely random fashion.

Owing to the linearity of the two branches of the tent maps, (5) is simplified as

$$
\rho_{n+1}(x)=\frac{\rho_{n}\left(y_{+}\right)+\rho_{n}\left(y_{-}\right)}{a(1-\epsilon)}
$$

where $y_{+}$and $y_{-}$are the two preimages of the same $x$, i.e. $x=F_{n}\left(y_{ \pm}\right)$.

In the following, we propose an expansion form of NFPE in order to facilitate the analysis of the model. Figure 11 shows snapshots of $\rho_{n}(x)$ for two parameter conditions. The density distributions for a turbulent state and a two-band state are displayed in Figs.1 $1 \mathrm{a}$ and [lb, respectively. We assume that the density distribution is a piecewise constant function, which is due to the piecewise linearity of the tent map. Then, one may simplify (8) by using a technique adopted to analyze a one-dimensional tent map [21]. The density distribution is expressed as

$$
\rho_{n}(x)=\sum_{j=0} b_{n}^{j} \theta\left(c_{n}^{j}-x\right),
$$

where $\theta(x)$ is a step function

$$
\theta(x)= \begin{cases}1 & (x \geq 0) \\ 0 & (x<0)\end{cases}
$$


In the above equation, $b_{n}^{j}$ and $c_{n}^{j}$ represent the height and the location of the jumps in $\rho_{n}(x)$, respectively. Since the initial condition for the density distribution is assumed uniform, $b_{1}^{j}$ and $c_{1}^{j}$ are given by

$$
\left\{\begin{array}{rlc}
b_{1}^{0} & = & \frac{1}{a(1-\epsilon)} \\
c_{1}^{0} & = & 1-\frac{a \epsilon}{2} \\
b_{1}^{1} & = & -\frac{1}{a(1-\epsilon)} \\
c_{1}^{1} & = & 1-\frac{a \epsilon}{2}-a(1-\epsilon) \\
\text { others } & = & 0
\end{array}\right.
$$

For $n=1$, the number of jumps in the density $\rho_{n}(x)$, which is denoted by $\nu$, equals two, and increases with $n$ as $\nu=n+1$. With the use of (8), the evolutional equations for $b_{n}^{j}$ and $c_{n}^{j}$ are given by

$$
\left\{\begin{array}{l}
b_{n+1}^{0}=\frac{2}{a(1-\epsilon)} \sum_{j^{\prime}=0} b_{n}^{j^{\prime}} \theta\left(c_{n}^{j^{\prime}}\right) \\
c_{n+1}^{0}=F_{n}(0) \\
b_{n+1}^{j+1}=-\frac{1}{a(1-\epsilon)} b_{n}^{j}\left(2 \theta\left(c_{n}^{j}\right)-1\right) \\
c_{n+1}^{j+1}=F_{n}\left(c_{n}^{j}\right)
\end{array}\right.
$$

This set of equations gives an alternative representation of NFPE (8). Note the identity

$$
\sum_{j=0} b_{n}^{j}=0
$$

which results from the requirement $\rho_{n}(-1)=0$. By using (雨) along with (9), the mean field $h_{n}$ is calculated as

$$
h_{n}=\left(1-\frac{a}{2} \sum_{j=0} b_{n}^{j} c_{n}^{j}\left|c_{n}^{j}\right|\right)
$$

Equations (6), (12) and (14) constitute a closed set of exact nonlinear equations equivalent to NFPE, and (11) specifies the initial condition.

The dimension of the dynamical system (12) is potentially infinite $(j=0,1, \ldots, \infty)$ because the number of jumps in $\rho_{n}(x)$ increases indefinitely with $n$. We now approximate 
this by a finite number. We expect that the jumps of sufficiently large $j$ hardly contribute to the collective behavior, so that we may ignore such minor jumps. Indeed, the third equation in (12) means that the height of the jumps is given by

$$
\left|b_{n}^{j}\right|=\frac{1}{a^{j}(1-\epsilon)^{j}}\left|b_{n-j}^{0}\right|
$$

Thus, $b_{n}^{j}$ approaches 0 exponentially as $j \rightarrow \infty$, provided $a(1-\epsilon)>1$. We now focus on this incoherent regime. Note that in the opposite case, i.e. $a(1-\epsilon)<1, \rho_{n}(x)$ becomes a delta function since all elements are in perfect synchrony, so that the breakdown of the above approximation is apparent. Our approximate method seems more convenient than those employed previously [13, 15]. In what follows, we restrict the maximum number of the jumps to 50 23]. The mean field behavior calculated with this approximation turns out consistent with our direct numerical simulation.

\section{STABILITY OF STATIONARY STATES}

We investigate stationary solutions i.e. fixed points of NFPE which are however not the fixed points of GCM (11). The fixed points of NFPE are determined by $\rho_{n+1}(x)=\rho_{n}(x) \equiv$ $\rho_{*}(x)$. From the expansion $(12)$ in the last section, this condition is given by

$$
\begin{aligned}
b_{*}^{0} & =\frac{2}{a(1-\epsilon)} \sum_{j^{\prime}=0} b_{*}^{j^{\prime}} \theta\left(c_{*}^{j^{\prime}}\right), \\
c_{*}^{0} & =F_{*}(0), \\
b_{*}^{j+1} & =-\frac{1}{a(1-\epsilon)} b_{*}^{j}\left(2 \theta\left(c_{*}^{j}\right)-1\right), \\
c_{*}^{j+1} & =F_{*}\left(c_{*}^{j}\right),
\end{aligned}
$$

where the map $F_{*}$ evaluated at the fixed point $\rho_{*}$ is given as

$$
\begin{aligned}
F_{*}(x) & =(1-\epsilon)(1-a|x|)+\epsilon h_{*}, \\
h_{*} & =\left(1-\frac{a}{2} \sum_{j=0} b_{*}^{j} c_{*}^{j}\left|c_{*}^{j}\right|\right) .
\end{aligned}
$$

The above equations constitute a set of self-consistent equations. For the time-being, we regard $h_{*}$ in (17) as an input field $h_{i n}$, and distinguish it from $h_{*}$ in (18) which we regard 
as an output field $h_{\text {out }}$. We can easily calculate $b_{*}^{j}$ and $c_{*}^{j}$ from (16) and the normalization condition for the density

$$
\sum_{j=0} b_{*}^{j}\left(c_{*}^{j}+1\right)=1
$$

With $b_{*}^{j}$ and $c_{*}^{j}$ thus obtained, the static field $h_{\text {out }}$ is given by (18). Consequently, $h_{\text {out }}$ is obtained as a function of $h_{\text {in }}$, i.e. $h_{\text {out }}\left(h_{\text {in }} ; a, e\right)$, which is called a "static mapping" [19]. The fixed points of this mapping give the stationary solutions of our system. The mapping actually has some fixed points in most of the parameter region under consideration.

Numerical stability analysis of the stationary states is now presented. We first linearize (12) about a stationary state $\left(b_{*}^{j}, c_{*}^{j}\right)$ corresponding to the density $\rho_{*}(x)$, and consider a small density deviation from it. Noting that $F_{n}(x)$ in (12) depends on $b_{n}^{j}$ and $c_{n}^{j}$, we have a set of linear maps given by

$$
\left\{\begin{array}{l}
\beta_{n+1}^{0}=\frac{2}{a(1-\epsilon)} \sum_{j^{\prime}=0} \beta_{n}^{j^{\prime}} \theta\left(c_{*}^{j^{\prime}}\right) \\
\gamma_{n+1}^{0}=-\frac{a \epsilon}{2} \sum_{j^{\prime}=0}\left\{\beta_{n}^{j^{\prime}} c_{*}^{j^{\prime}}\left|c_{*}^{j^{\prime}}\right|+2 \gamma_{n}^{j^{\prime}} b_{*}^{j^{\prime}}\left|c_{*}^{j^{\prime}}\right|\right\} \\
\beta_{n+1}^{j+1}=-\frac{1}{a(1-\epsilon)} \beta_{n}^{j}\left(2 \theta\left(c_{*}^{j}\right)-1\right) \\
\gamma_{n+1}^{j+1}=-\frac{a \epsilon}{2} \sum_{j^{\prime}=0}\left\{\beta_{n}^{j^{\prime}} c_{*}^{j^{\prime}}\left|c_{*}^{j^{\prime}}\right|+2 \gamma_{n}^{j^{\prime}} b_{*}^{j^{\prime}}\left|c_{*}^{j^{\prime}}\right|\right\}-a(1-\epsilon) \gamma_{n}^{j}\left(2 \theta\left(c_{*}^{j}\right)-1\right)
\end{array}\right.
$$

where $\beta_{n}^{j}=b_{n}^{j}-b_{*}^{j}$ and $\gamma_{n}^{j}=c_{n}^{j}-c_{*}^{j}$. The set of equations above can be expressed with a coefficient matrix $D$ as

$$
\left(\begin{array}{l}
\vec{\beta}_{n+1} \\
\vec{\gamma}_{n+1}
\end{array}\right)=D\left(\begin{array}{l}
\vec{\beta}_{n} \\
\vec{\gamma}_{n}
\end{array}\right) .
$$

The eigenvalues of $D$ determines the stability of the stationary solution. It is obvious from (20) that $D$ has the form

$$
D=\left(\begin{array}{cc}
D_{1} & 0 \\
* & D_{2}
\end{array}\right) .
$$

Thus, the eigenvalues of $D$ are given by those of $D_{1}$ and $D_{2}$, and they are generally complex numbers. In Fig.2, we show the eigenvalues of $D$ for $a=1.5$ and $\epsilon=0.05$ for which a 
stationary state exists stably. They include real numbers and complex-conjugate pairs, and their distribution is roughly given by two concentric circles. The inner and outer circles correspond to $D_{1}$ and $D_{2}$, respectively. The normalization condition for $\rho_{n}(x)$ requires that one of the eigenvalues is always unity [14]. If all of the remaining eigenvalues lie within the unit circle, the stationary solution remains stable. It loses stability in the following two ways.

The first case is due to band splitting. This occurs at $a(1-\epsilon)=\sqrt{2}$ [24]. In the unstable region, period-two motion appears, and then the density $\rho_{n}(x)$ has a two-band structure as shown in Fig.1]b. An infinite number of period-two solutions exist and a particular state is selected out by imposing a specific initial condition. Therefore, it is expected that their stability is marginal.

Figure 3 a shows the eigenvalues under the condition that stationary state is stable $(a(1-$ $\epsilon)<\sqrt{2}$ ). The moduli of all eigenvalues except for one with vanishing argument are smaller than unity. Figure $3 \mathrm{~b}$ shows the eigenvalues after band splitting $(a(1-\epsilon)>\sqrt{2})$. The matrix $D_{1}$ has two eigenvalues with moduli 1 , while their arguments are given by to 0 and $\pi$ (the eigenvalues are 1 and -1 , respectively). In Fig. 田, we plotted the eigenvalue $\lambda_{\pi}$ with argument $\pi$ as a function of $\epsilon$. As is seen from Fig. $4 \mathrm{a} a,\left|\lambda_{\pi}\right|$ approaches unity as $\epsilon$ increases up to the band-splitting point $\epsilon_{0}$ under fixed $a$ at 1.5. For this value of $a$, the band splitting is seen for $\epsilon>\epsilon_{0}=\frac{3-2 \sqrt{2}}{3}=0.05719 \cdots$, and $\left|\lambda_{\pi}\right|$ is kept at unity over this range of $\epsilon$. The results of the stability analysis agree with our observation from direct numerical simulation. In order to investigate the nature of this instability in some detail, we make a log-log plot near the band-splitting point. This is shown in Fig.4 linearly with $\epsilon_{0}-\epsilon$. Thus, the band-splitting point can be determined accurately from this kind of analysis.

There is another type of instability. This is associated with a Hopf bifurcation, and occurs even in the region where the band splitting is not seen $(a(1-\epsilon)<\sqrt{2})$. Figure $3 \mathrm{c}$ shows the distribution of the eigenvalues in the unstable regime. $\left|\lambda_{\pi}\right|$ is smaller than unity and the matrix $D_{2}$ has an eigenvalue with modulus larger than unity. The argument of 
this eigenvalue is about $0.86 \pi$. In this region, the collective behavior of the original system is found quasiperiodic. The evolution of the corresponding mean field $h_{n}$ is displayed in Fig.5, and its power spectrum is given in Fig.6. The latter has some sharp peaks, and the frequency of the highest peak is about 0.43 . This value is consistent with the results of our stability analysis.

\section{SUMMARY}

The piecewise-linearity of the map allows us to make an expansion of NFPE in term of the height and location of the jumps in the density distribution. We analyzed the stability of stationary solutions by using this expansion. Linearization of the expansion is expressed with

two matrices $D_{1}$ and $D_{2}$. The band-splitting instability is associated with the eigenvalues of $D_{1}$. Stability analysis predicts the band-splitting point accurately. The band splitting results in marginally stable period-two states. There is also a region where quasi-periodic motions appear. The corresponding instability is essentially a Hopf bifurcation, and associated with the eigenvalues of $D_{2}$.

Our analysis presupposes infinite system size. In actual finite system, however, we expect some fluctuations around the solutions of NFPE.

We investigated only globally coupled tent maps. It should be mentioned, however, that the same methods could also be useful for investigating other piecewise linear maps.

\section{ACKNOWLEDGMENTS}

The author would like to acknowledge valuable advices from Y.Kuramoto and fruitful discussions with T.Chawanya and the members of Nonlinear Dynamics group of Kyoto University. 


\section{REFERENCES}

[1] K. Wiesenfeld and P. Hadley, Phys. Rev. Lett. 62 (1989) 1335.

[2] K. Wiesenfeld, C. Bracikowski, G. James and R. Roy, Phys. Rev. Lett. 65 (1990) 1749.

[3] S.H. Strogatz, C.M. Marcus, R.M. Westervelt and R.E. Mirollo, Physica D 36 (1989) 23.

[4] T. Ikegami and K. Kaneko, chaos 2 (1992) 397.

[5] H. Somplinsky, D. Golomb and D. Kleinfeld, Phys. Rev. A 43 (1991) 6990.

[6] D. Golomb, D. Hansel, B.Sharaiman and H. Sompolinsky, Phys. Rev. A 45 (1992) 3516.

[7] K. Kaneko, Physica D 77 (1994) 456.

[8] H. Nozawa, chaos 2 (1992) 377.

[9] Y. Kuramoto, Prog. Theor. Phys. Suppl. 79 (1984) 223.

[10] K. Kaneko, Physica D 34 (1989) 1.

[11] H. Chaté and P. Manneville, Europhys. Lett. 17 (1992) 291; Prog. Theor. Phys. 87 (1992) 1.

[12] K. Kaneko, Physica D 41 (1990) 137.

[13] K. Kaneko, Physica D 55 (1992) 368.

[14] W. Just, J. Stat. Phys. 79 (1995) 429; Physica D 81 (1995)317.

[15] A.S. Pikovsky and J. Kurths, Physica D 76 (1994) 411; Phys. Rev. Lett. 72 (1994) 1644.

[16] K. Kaneko, Phys. Rev. Lett. 65, (1990) 1391.

[17] K. Kaneko, Proceeding of the "Gran Finale" conference, Como (1993).

[18] G. Perez, S. Sinha and A. Cerdeira, Physica D 63 (1993) 341. 
[19] G. Perez and A. Cerdeira, Phys. Rev. A 46 (1992) 7492.

[20] S. Sinha, D. Biswas, M. Azam, and S.V. Lawande, Phys. Rev. A 46, (1992) 6242; Phys. Rev. Lett. 69 (1992) 3306.

[21] M. Dörfle, J. Stat. Phys. 40 (1985) 93.

[22] M. Ding and L.T. Wille, Phys. Rev. E 48 (1993) R1608.

[23] The parameters we used satisfy $a(1-\epsilon) \simeq \sqrt{2}$. Therefore the height of the jumps ignored is not larger than $2^{-25}$.

[24] Higher order band splitting is also seen. At the critical parameter values $a(1-\epsilon)=2^{1 / 2^{n}}$, $2^{n}$-band structure appears. The period of this state is $2^{n}$. If the population in some bands is zero, the number of bands can be smaller than $2^{n}$. 


\section{FIGURES}

FIG. 1. Snapshot of the density distribution $\rho_{n}(x)$ after transients have subsided. (a) Turbulent state obtained from the expansion of NPFE (see Section 2); $a=1.6, \epsilon=0.05$. (b) Two-band state; $a=1.6, \epsilon=0.15$.

FIG. 2. Eigenvalues of the $50 \times 50$ matrices $D_{1}$ and $D_{2}$ for $a=1.5$ and $\epsilon=0.05$ where the stationary solution remains stable.

FIG. 3. Eigenvalues of the matrices $D_{1}$ and $D_{2}$. Their absolute values vs. arguments are shown. Since the eigenvalues appear as complex-conjugate pairs, only those with arguments lying between 0 and (a) $a=1.5, \epsilon=0.05$. The moduli of all eigenvalues are smaller than 1 except one with vanishing argument. (b) $a=1.5, \epsilon=0.06$. There exists an eigenvalue of modulus 1 and argument $\pi$. (c) $a=1.9, \epsilon=0.12$. There exists an eigenvalue with modulus is larger than 1 .

FIG. 4. (a) Eigenvalue $\left|\lambda_{\pi}\right|$ with argument $\pi$ vs. $\epsilon$ where $a=1.5$. (b) Log-log plot near the band-splitting point $\epsilon_{0}$ where $a=1.5$.

FIG. 5. Trajectory of the mean field $h_{n}$ obtained from the expansion of NPFE (see section 2). The parameter values are the same as in Fig.2c (i.e. $a=1.9, \epsilon=0.12$ ). This was obtained from 1000 steps after transients have subsided.

FIG. 6. Power spectrum of the time series of the mean field $h_{n}$ obtained from the expansion of NPFE (see section 2). This was obtained from an average over 10 runs each of 1024 iterations after transients have subsided. Parameter values are the same as in Fig.2c. 


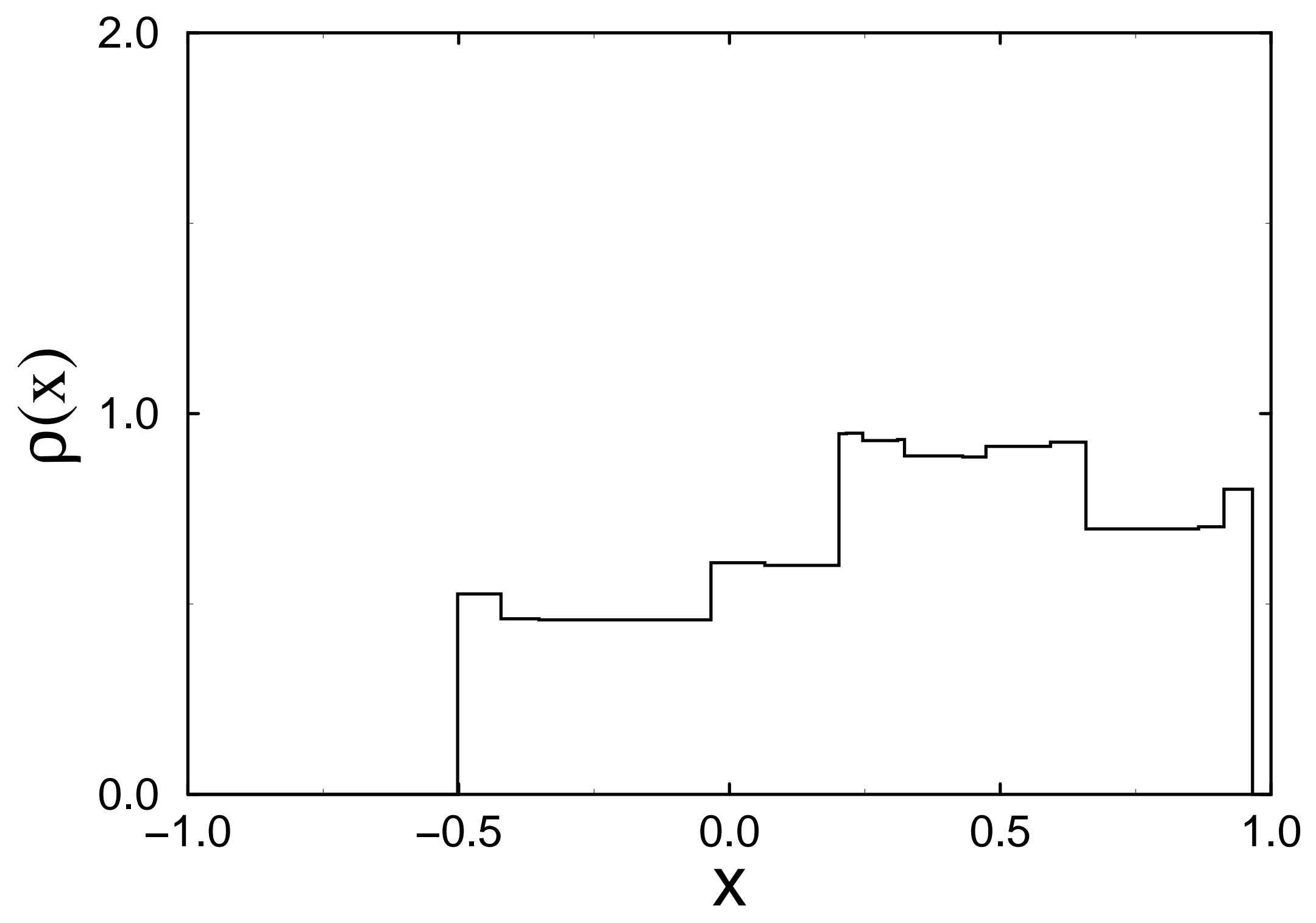




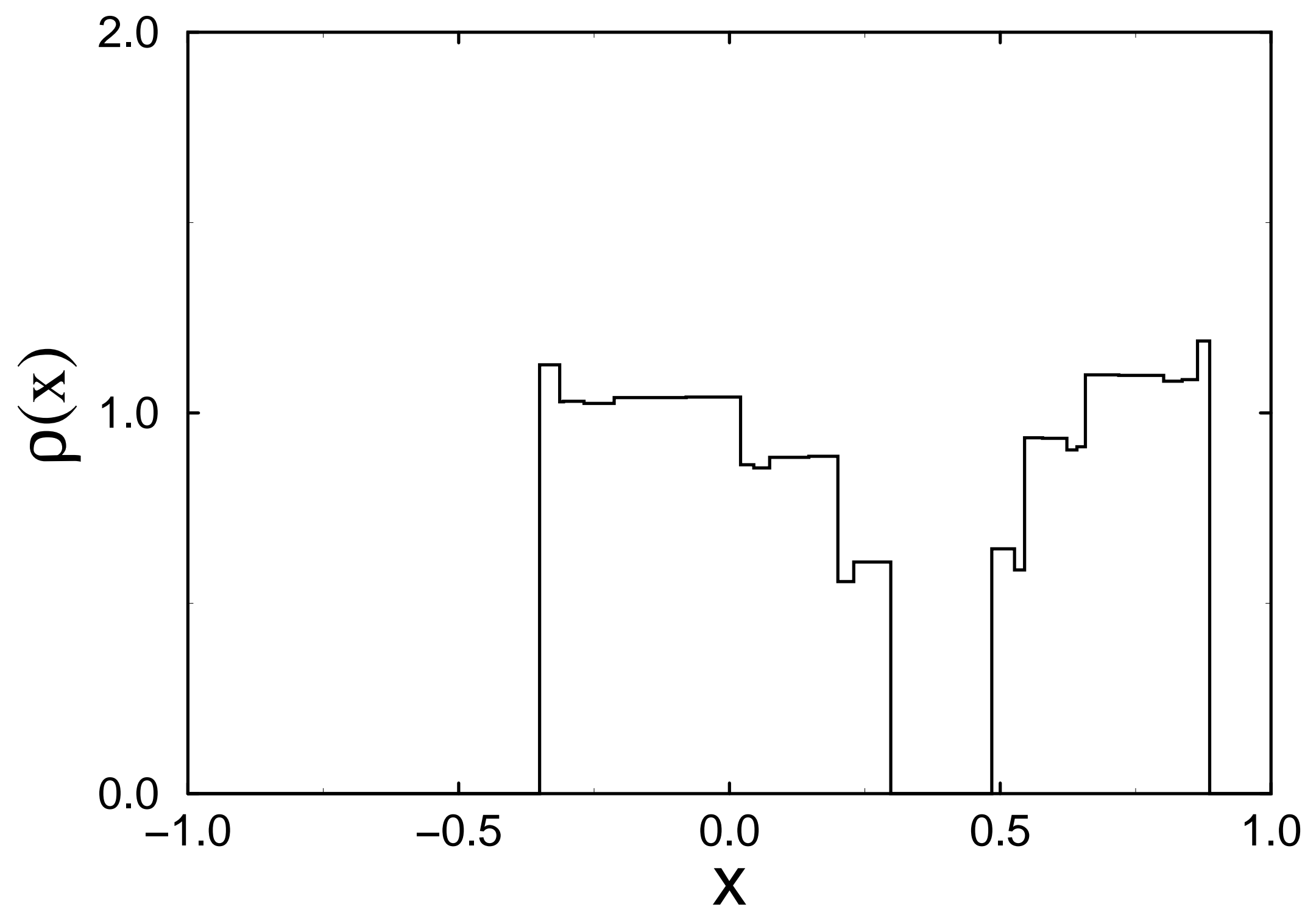




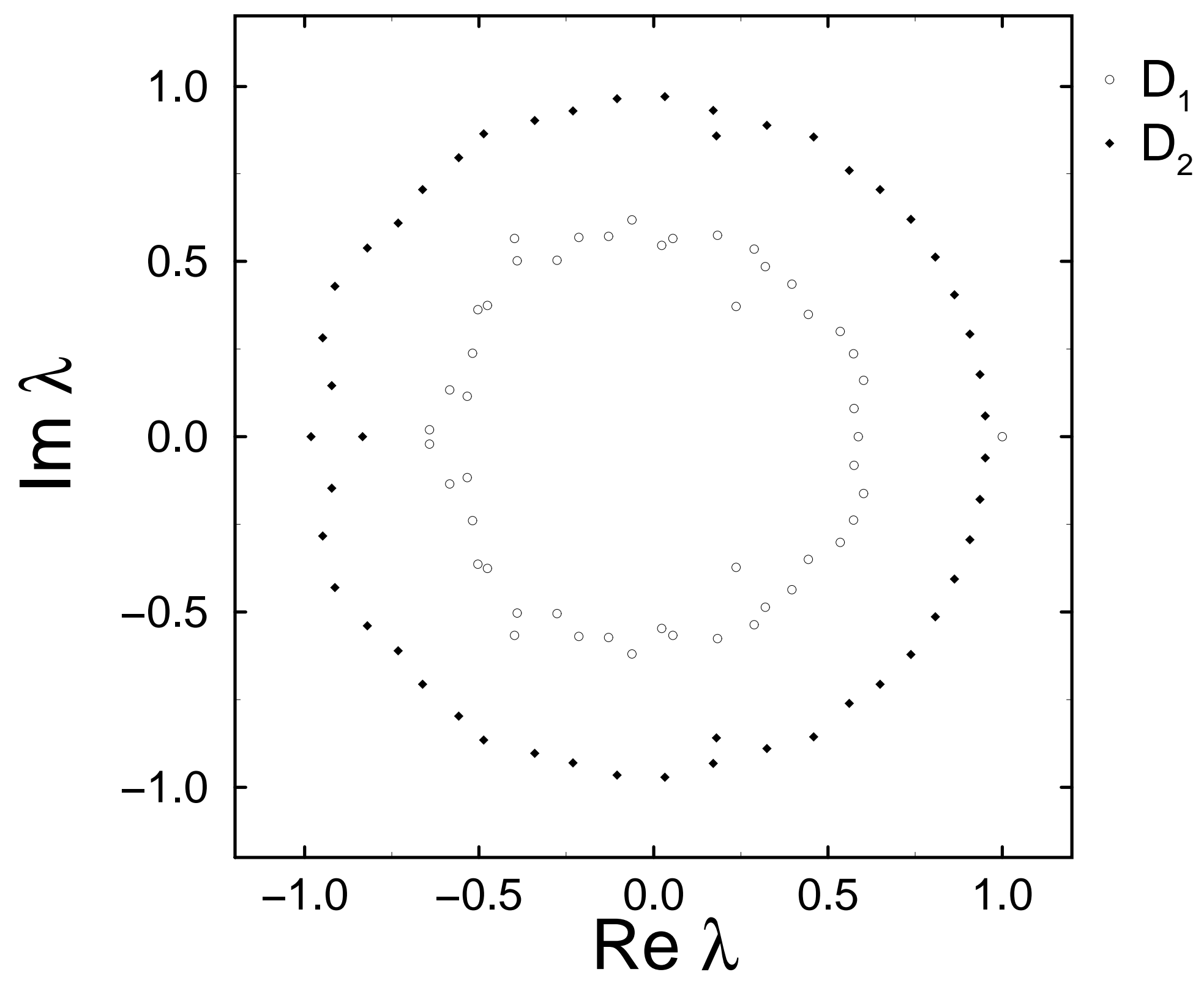




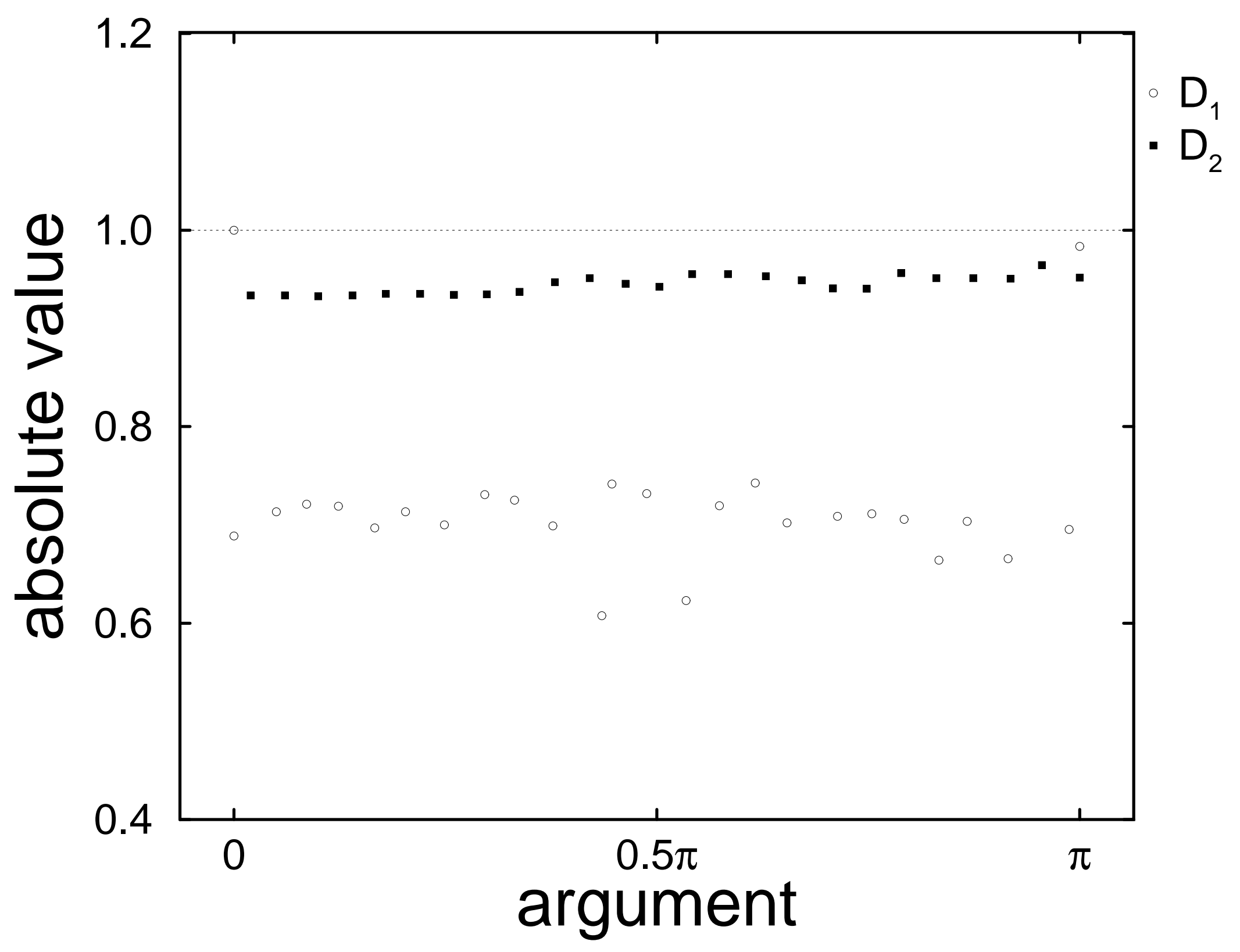




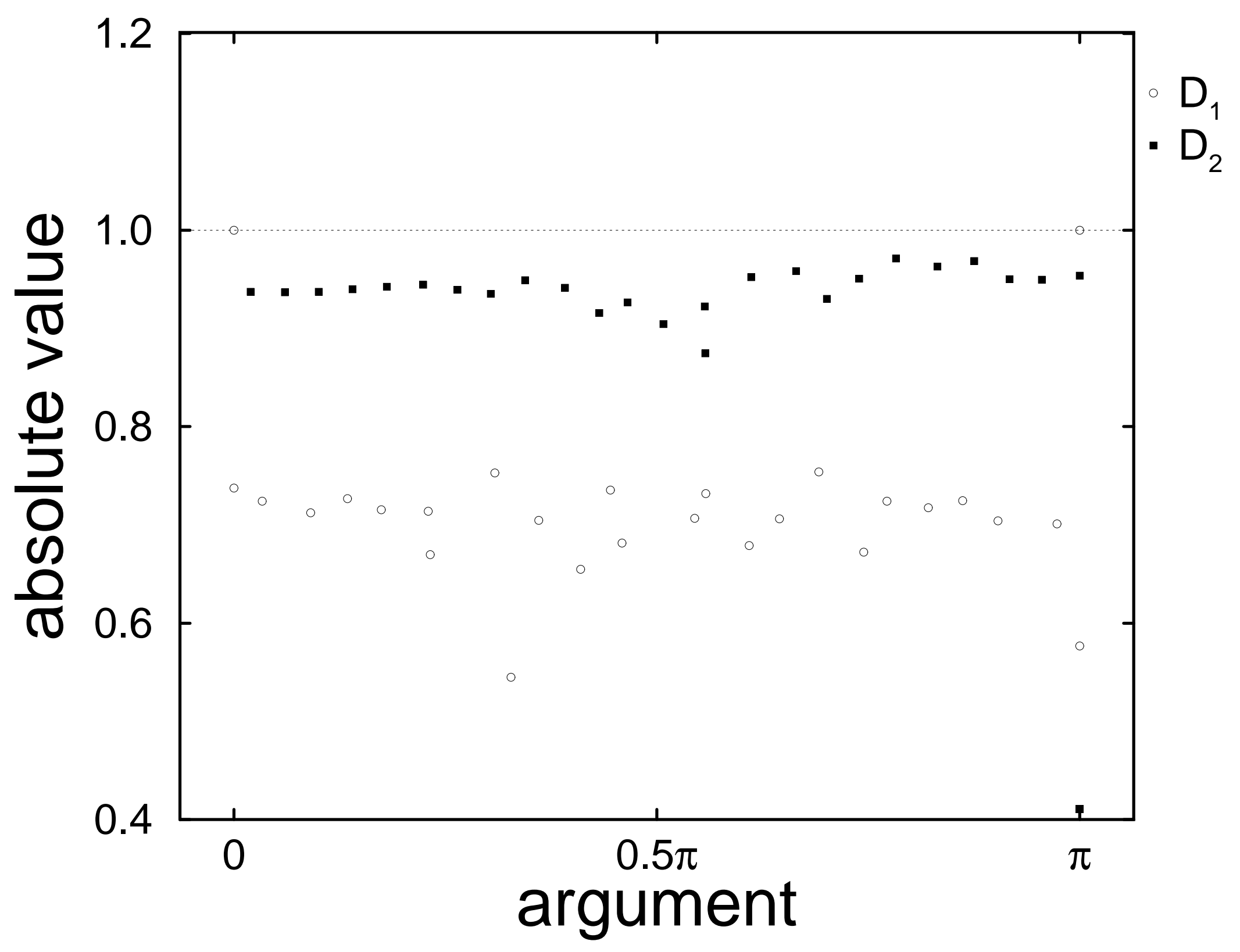




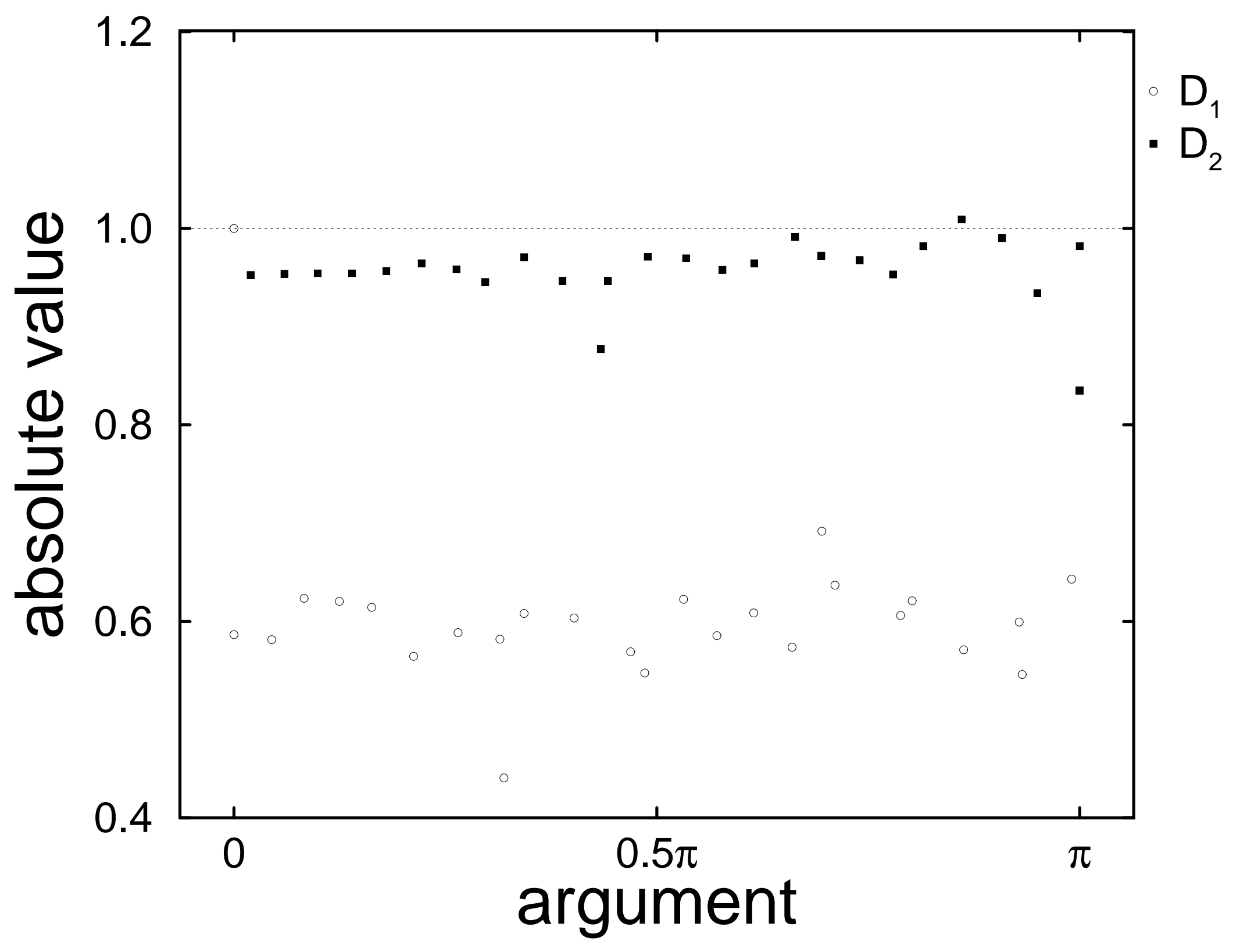




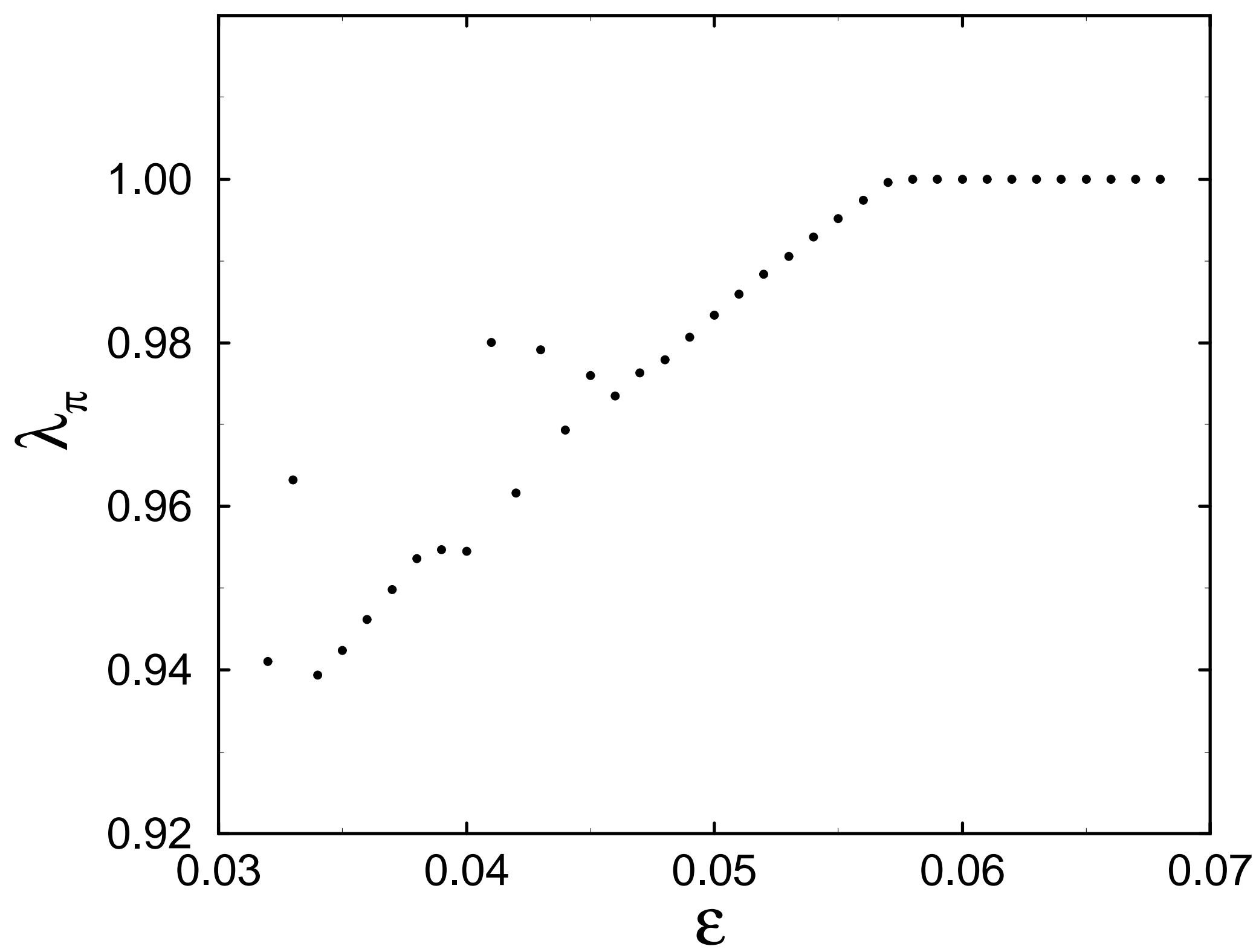




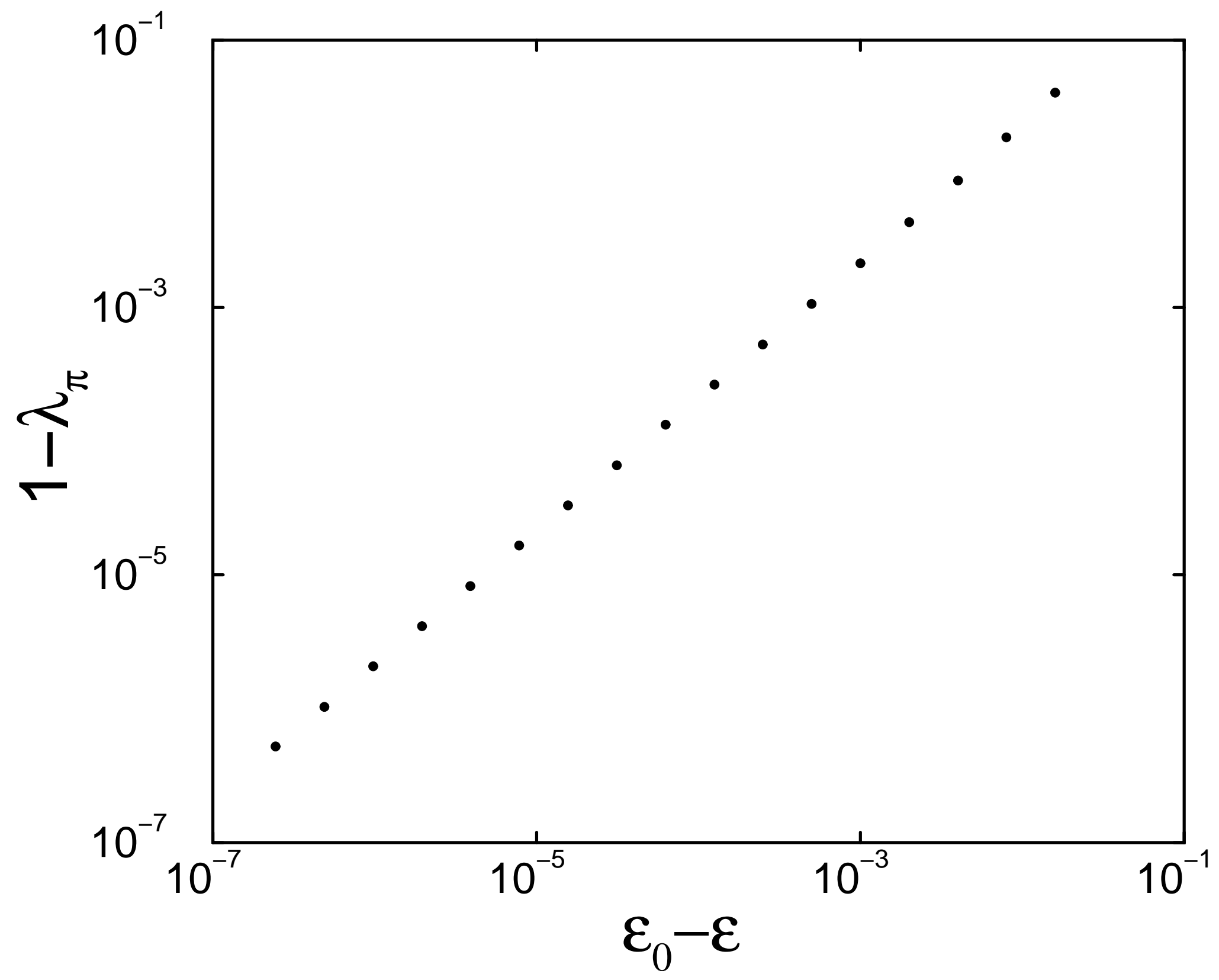




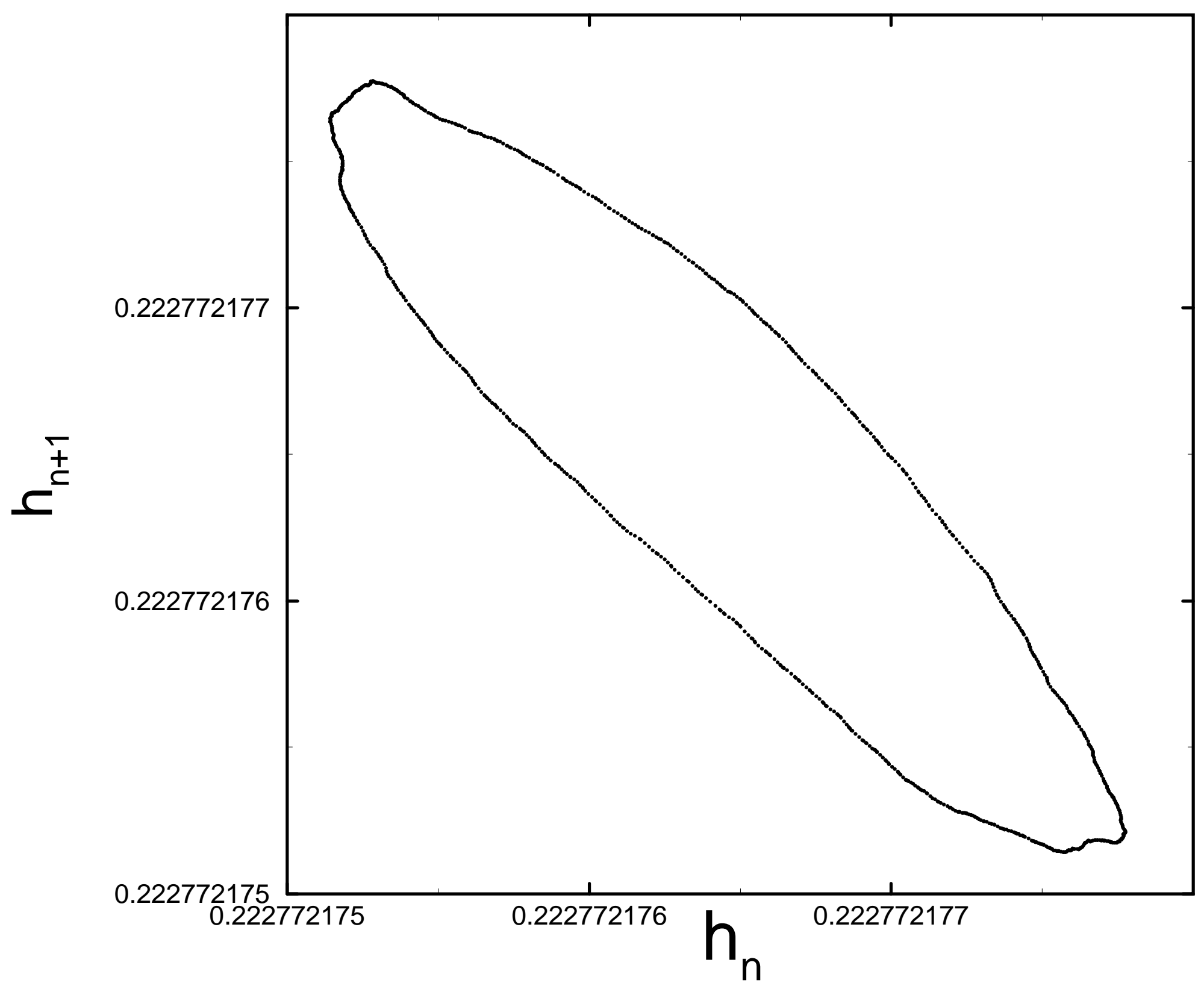




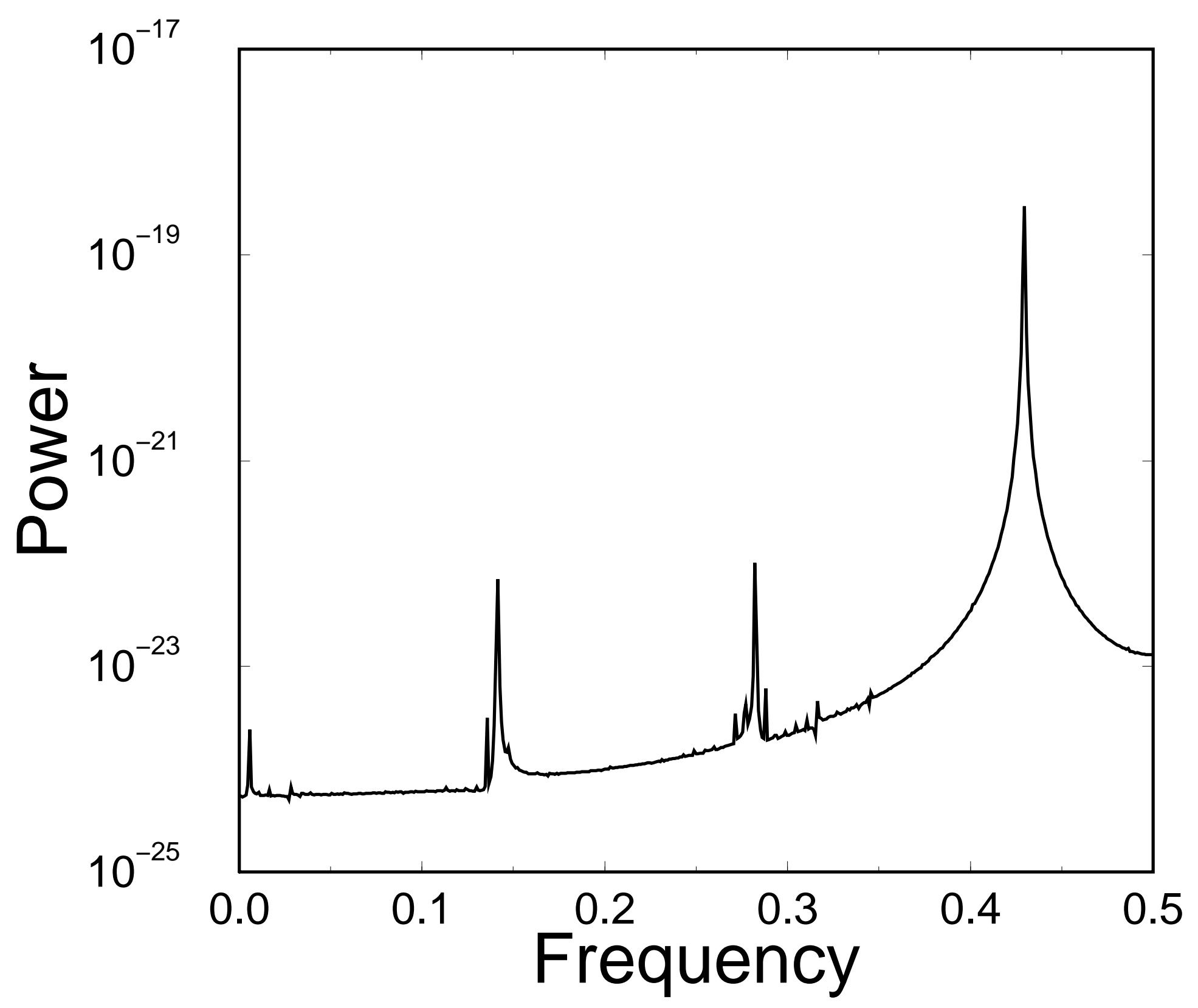

